Pumping a photoionization atomic inner-shell $x$-ray laser with $x$-ray free-electron laser radiation

N. Rohringer, R. London

July 20, 2009

SPIE Optics and Photoniccs, Soft X-ray lasers and applications, VIII

San Diego, CA, United States

August 4, 2009 through August 6, 2009 
This document was prepared as an account of work sponsored by an agency of the United States government. Neither the United States government nor Lawrence Livermore National Security, LLC, nor any of their employees makes any warranty, expressed or implied, or assumes any legal liability or responsibility for the accuracy, completeness, or usefulness of any information, apparatus, product, or process disclosed, or represents that its use would not infringe privately owned rights. Reference herein to any specific commercial product, process, or service by trade name, trademark, manufacturer, or otherwise does not necessarily constitute or imply its endorsement, recommendation, or favoring by the United States government or Lawrence Livermore National Security, LLC. The views and opinions of authors expressed herein do not necessarily state or reflect those of the United States government or Lawrence Livermore National Security, LLC, and shall not be used for advertising or product endorsement purposes. 


\title{
Pumping a photoionization atomic inner-shell x-ray laser with $\mathrm{x}$-ray free-electron laser radiation
}

\author{
Nina Rohringer and Richard London \\ Lawrence Livermore National Laboratory, Livermore, California 94551, USA
}

\begin{abstract}
Focusing an x-ray free electron laser (XFEL) pulse into a gas target, a plasma of transiently core excited ions can be created within a few fs, building a pathway to an inner-shell keV x-ray laser. Varying the XFEL parameters, a wide variety of pulse structures can be created with comparable peak-intensities to XFELs: isolated pulses of subfs duration, trains of pulses with increased temporal coherence, and trains of fs pulses of different wavelengths. We present self-consistent gain and amplification calculations, tailored to predict first experiments on lasing in neon pumped by the Linac Coherent Light Source at Stanford.
\end{abstract}

Keywords: X-ray free electron laser, inner-shell atomic x-ray laser

\section{INTRODUCTION}

The first demonstration of a laboratory atomic x-ray laser (XRL) dates back 25 years. ${ }^{1}$ Since then, a world-wide community has worked to advance the parameters and applications of these sources. The shortest wavelength achieved so far with an atomic XRL is $3.6 \mathrm{~nm}$, albeit at a low output intensity. ${ }^{2}$ Recently, lasing of the first $\mathrm{x}$-ray free electron laser (XFEL) - the Linac Coherent Light Source (LCLS) at Stanford ${ }^{3}$ - was demonstrated at $8 \mathrm{keV}$ photon energy. ${ }^{4}$ We propose to amalgamate these two technologies. In this contribution we give a theoretical case study, exploring the possibility to pump an inner-shell atomic x-ray laser with an XFEL. This will open the pathway to a wide variety of x-ray pulse structures with peak intensities comparable to those of the pumping XFEL and wavelengths of $1.5 \mathrm{~nm}$ or less. We discuss the results of a theoretical investigation showing that it might be possible to isolate single pulses of sub-femtosecond duration, to create a train of pulses of improved temporal coherence and to produce a series of temporally separated femtosecond pulses of different wavelengths. This output is suitable for applications in multi-color x-ray pump-probe experiments.

Forthcoming XFEL sources will be based on the self-amplified spontaneous emission (SASE) process. ${ }^{5,6}$ This process creates pulses with chaotic and spiky temporal and spectral intensity profile. The created pulses can be seen as an ensemble of uncorrelated intensity spikes of femtosecond duration, of random width, height, position and phase. Typical coherence times of such sources are 2-3 fs at $1 \mathrm{keV}$ photon energy. The high peak brilliance of SASE XFELs will open the pathway to study non-linear optical processes in the x-ray regime for the first time. These processes depend on higher order coherence properties of the XFEL, which makes the interpretation of experimental results and determining fundamental cross-sections for non-linear optical processes a delicate, difficult task. ${ }^{7-13}$ Control of the pulse duration and isolating single x-ray pulses of femtosecond duration would be an advantage in interpreting the results of multi-photon processes in the x-ray regime. Our proposed atomic XRL scheme is a first step into this direction and could be realized in the near future.

The quest for ultrashort high-intensity x-ray sources is being tackled with several techniques. Proposals to produce fs x-ray pulses at XFEL facilities are based on slicing of the electron beam ${ }^{14}$ or operation with lowcharge, ultra-low emittance electron beams. ${ }^{15}$ Lately, a scheme to produce a train of attosecond pulses by mode locking of an XFEL was proposed. ${ }^{16}$ At the other end of the spectrum are optical laser pumped XRLs seeded with high-harmonic radiation. ${ }^{17}$ Due to the lack of high-harmonic radiation at shorter wavelength, this method is currently limited to the soft x-ray regime $(>10 \mathrm{~nm})$. Recently, the idea of using an FEL to pump a laser in the XUV regime was put forward for an inner shell scheme in carbon ${ }^{18}$ and a recombination scheme in helium. ${ }^{19}$ Another approach aims at pumping an XRL with broadband betatron radiation. ${ }^{20}$ We propose a lasing scheme of broad capability, by photo-pumping an inner-shell XRL ${ }^{21}$ with an XFEL. The method can be applied to a broad variety of gain materials. In this contribution we present gain calculations for neon, to predict first lasing experiments using the LCLS.

The article is organized as follows: in the next section we describe the lasing scheme, then we discuss results 
of small-signal gain calculations at different photon pumping energies in section 3 . In section 4 , we present a one-dimensional self-consistent gain model to determine the X-ray laser output and show results for a typical SASE sample pump pulse.

\section{DESCRIPTION OF THE INNER-SHELL LASING SCHEME}

The basic idea is to use an XFEL to create a population inversion by inner-shell photoionization. The concept of a photoionization-pumped x-ray laser is not new, ${ }^{21,22}$ but due to the lack of appropriate x-ray pumping sources, it could not be experimentally realized so far. Focusing an XFEL into a gas target, an elongated plasma column of core-excited ions is created by inner-shell photoionization within the first few femtoseconds of the XFEL pulse. ${ }^{13}$ The core-excited ions relax by either Auger decay or radiative decay. In case of neon, the Auger lifetime of a K-shell hole is 2.7 femtoseconds, therefore allowing a transient population inversion of only femtosecond duration. This ultra-short lived population inversion forms the basis of the x-ray transition. Since the inner-shell photoionization and build-up of population inversion happens on an ultrafast time scale, i.e. on a time scale short to typical ion-electron collisions in a gas target of moderate density, the ion temperature in the plasma column is expected to remain cold. The line width of the x-ray lasing transition is then dominated by the Auger width of the core-hole, which opens the pathway to very narrow high-gain lasing transitions. The proposed lasing scheme is self-terminating, i.e. each atom can at most contribute one photon of a given transition during the amplification process. The lower lasing state in our pumping scheme is efficiently depleted by another innershell photoionization event. This is in stark contrast to recently proposed XUV FEL pumped lasing schemes using inner valence electrons, ${ }^{18}$ where depletion of the lower lasing level is caused by Coster-Kronig transitions, i.e. lasing occurs between two autoionizing states. Our scheme is widely applicable, by tuning the pumping photon energy above the core-ionization edge of the lower lying lasing state and can be extended to any other atomic species.

The geometry of the XRL is determined by the focus of the XFEL. Focusing an XFEL pulse of $1 \mathrm{keV}$ photon energy with a Gaussian spatial beam profile to a focal radius of $r=1 \mu \mathrm{m}$ results in a focal depth of approximately $5 \mathrm{~mm}$, which defines the length $L$ of the amplifying plasma column of extremely low aspect ratio. The spatial coherence properties of the XRL can be characterized by the geometry of the x-ray amplifying plasma column ${ }^{23}$ : The transverse coherence length is given by $L_{T}=\frac{\lambda L}{2 \pi r}$. For a lasing transition around $\lambda=1 \mathrm{~nm}$, we therefore find a transverse coherence length of $L_{T}=1 \mu \mathrm{m}$, implying that the XRL will have nearly full spatial coherence.

\section{SMALL SIGNAL GAIN CALCULATIONS}

In a simple one-dimensional gain model, amplification of radiation in the exponential gain regime is governed by

$$
j(z, t)=j(0, t) \cdot e^{n \cdot z \cdot g(t)}
$$

where $j(z, t)$ denotes the flux at point $z$ as a function of time $t$ (to describe the temporal pulse structure), $\mathrm{j}(0, \mathrm{t})$ is the seed (in our case, spontaneous emission), $n$ is the density of the gain material and $g(t)$ is the so-called small-signal gain cross section, which is given by

$$
g(t)=n_{U}(t) \sigma_{s t i m}-n_{L}(t) \sigma_{a b s} .
$$

The small-signal gain cross section is determined by the occupancies of the upper and lower lasing states $n_{U}$ and $n_{L}$, respectively and by the cross-sections $\sigma_{\text {stim }}$ and $\sigma_{a b s}$ for stimulated emission and absorption,

$$
\sigma_{s t i m}=A_{U \rightarrow L} \frac{2 \pi c^{2}}{\omega^{2} \Delta \omega}, \quad \sigma_{a b s}=\sigma_{s t i m} \frac{g_{U}}{g_{L}},
$$

where $A_{U \rightarrow L}$ is the Einstein A coefficient for the radiative transition and $g_{U}$ and $g_{L}$ are the statistical weights of the upper and lower lasing levels. Eq. (3) gives the cross sections at the peak of the line, supposing a Lorentzian line shape. The line width of the transition $\Delta \omega$ is dominated by the total lifetime of the upper and lower states (Auger-life time and radiative lifetime). This is in contrast to traditional optical-laser pumped atomic x-ray lasers, where the line width is determined by Doppler and Stark broadening for high density plasmas. In our proposed x-ray laser scheme the amplifying plasma column is cold (close to room temperature) since the 
plasma and population inversion is created directly by the pumping XFEL on an ultra-short time scale. This time scale is 5-10 fs which is small compared to typical ion-electron coupling times at moderate gas densities*. Therefore, collisional broadening and collisional heating of the ions by hot photo and Auger electrons can be ruled out; Doppler broadening at room temperature is negligible compared to the natural line width of the lasing transitions. The relative natural line widths of x-ray lasing transitions in neon of different charge states are listed in Tables 1 and 2. This ultrafast pumping scheme, hence, establishes a basis for small-bandwidth, high-gain x-ray lasing transitions.

The level occupancies $n_{U}$ and $n_{L}$ of Eq. (2) are determined by the influence of the XFEL pump radiation

Table 1. Saturation intensity, average of the peak value of small-signal gains and the duration (FWHM) in fs and their standard deviation for an ensemble of 10,000 random pulses for $\omega_{P}=1 \mathrm{keV}$. The pulse parameters correspond to those of Fig.1.

\begin{tabular}{ccccccccc}
\hline Ne & upper state & $\omega[\mathrm{eV}]$ & $\Delta \omega / \omega$ & gain [a.u.] & $\mathrm{STD}_{\text {gain }}$ & $\tau[\mathrm{fs}]$ & $\mathrm{STD}_{\tau}$ & $I_{\text {sat }}\left[\mathrm{W} / \mathrm{cm}^{2}\right]$ \\
\hline \hline $1+$ & $1 \mathrm{~s}^{1} 2 \mathrm{~s}^{2} 2 \mathrm{p}^{6}$ & 849.8 & $2.9 \times 10^{-4}$ & 0.564 & 0.102 & 4.3 & 1.9 & $9.3 \times 10^{14}$ \\
$2+$ & $2 \mathrm{~s}^{2} 2 \mathrm{p}^{6}$ & 946.5 & $7.6 \times 10^{-4}$ & 0.107 & 0.061 & 2.6 & 1.0 & $3.4 \times 10^{15}$ \\
$3+$ & $1 \mathrm{~s}^{1} 2 \mathrm{p}^{6}$ & 863.1 & $2.5 \times 10^{-4}$ & 0.064 & 0.017 & 5.7 & 2.2 & $6.3 \times 10^{14}$ \\
$3+$ & $1 \mathrm{~s}^{1} 2 \mathrm{~s}^{2} 2 \mathrm{p}^{4}$ & 861.9 & $2.1 \times 10^{-4}$ & 0.422 & 0.105 & 6.9 & 2.5 & $6.6 \times 10^{14}$ \\
$3+$ & $1 \mathrm{~s}^{1} 2 \mathrm{~s}^{1} 2 \mathrm{p}^{5}$ & 862.3 & $2.2 \times 10^{-4}$ & 0.179 & 0.058 & 4.6 & 1.9 & $5.6 \times 10^{14}$ \\
\hline
\end{tabular}

Table 2. Same as in Table 1 but for $\omega_{P}=1.4 \mathrm{keV}$.

\begin{tabular}{ccccccccc}
\hline $\mathrm{Ne}$ & upper state & $\omega[\mathrm{eV}]$ & $\Delta \omega / \omega$ & gain $[$ a.u. $]$ & $\mathrm{STD}_{\text {gain }}$ & $\tau[\mathrm{fs}]$ & $\mathrm{STD}_{\tau}$ & $I_{\text {sat }}\left[\mathrm{W} / \mathrm{cm}^{2}\right]$ \\
\hline \hline $1+$ & $1 \mathrm{~s}^{1} 2 \mathrm{~s}^{2} 2 \mathrm{p}^{6}$ & 849.8 & $2.9 \times 10^{-4}$ & 0.466 & 0.101 & 5.3 & 2.4 & $9.3 \times 10^{14}$ \\
$2+$ & $2 \mathrm{~s}^{2} 2 \mathrm{p}^{6}$ & 946.5 & $7.6 \times 10^{-4}$ & 0.051 & 0.032 & 2.3 & 1.1 & $3.4 \times 10^{15}$ \\
$3+$ & $1 \mathrm{~s}^{1} 2 \mathrm{~s}^{2} 2 \mathrm{p}^{4}$ & 861.9 & $2.1 \times 10^{-4}$ & 0.199 & 0.041 & 7.2 & 3.2 & $6.6 \times 10^{14}$ \\
$3+$ & $1 \mathrm{~s}^{1} 2 \mathrm{~s}^{1} 2 \mathrm{p}^{5}$ & 862.3 & $2.2 \times 10^{-4}$ & 0.061 & 0.023 & 3.4 & 1.5 & $5.6 \times 10^{14}$ \\
$5+$ & $1 \mathrm{~s}^{1} 2 \mathrm{p}^{4}$ & 879.8 & $1.4 \times 10^{-4}$ & 0.067 & 0.022 & 4.0 & 2.0 & $2.6 \times 10^{14}$ \\
$5+$ & $1 \mathrm{~s}^{1} 2 \mathrm{~s}^{1} 2 \mathrm{p}^{3}$ & 879.9 & $1.1 \times 10^{-4}$ & 0.126 & 0.040 & 4.4 & 2.3 & $2.0 \times 10^{14}$ \\
$5+$ & $1 \mathrm{~s}^{1} 2 \mathrm{~s}^{2} 2 \mathrm{p}^{2}$ & 880.4 & $1.2 \times 10^{-4}$ & 0.092 & 0.015 & 10.9 & 4.9 & $3.5 \times 10^{14}$ \\
$7+$ & $1 \mathrm{~s}^{1} 2 \mathrm{p}^{2}$ & 902.6 & $3.2 \times 10^{-5}$ & 0.351 & 0.090 & 5.9 & 4.2 & $2.6 \times 10^{13}$ \\
$7+$ & $1 \mathrm{~s}^{1} 2 \mathrm{~s}^{1} 2 \mathrm{p}^{1}$ & 903.7 & $1.6 \times 10^{-5}$ & 0.506 & 0.042 & 26.3 & 7.6 & $1.2 \times 10^{13}$ \\
$8+$ & $1 \mathrm{~s}^{1} 2 \mathrm{p}^{1}$ & 916.4 & $1.5 \times 10^{-6}$ & 0.364 & 0.198 & 39.8 & 28.1 & $1.1 \times 10^{11}$ \\
$8+$ & $2 \mathrm{p}^{2}$ & 1006.9 & $7.9 \times 10^{-5}$ & 0.698 & 0.234 & 12.7 & 12.4 & $8.3 \times 10^{13}$ \\
$8+$ & $2 \mathrm{~s}^{1} 2 \mathrm{p}^{1}$ & 1008.6 & $3.5 \times 10^{-5}$ & 0.896 & 0.127 & 40.6 & 24.3 & $3.6 \times 10^{13}$ \\
$9+$ & $2 \mathrm{p}^{1}$ & 1022.0 & $4.0 \times 10^{-6}$ & 6.131 & 2.718 & 14.9 & 12.2 & $4.4 \times 10^{11}$ \\
\hline
\end{tabular}

on an ensemble of single atoms. The occupancies for different configuration states at different charge states are calculated by solving a system of rate equations, describing valence and core photoionization, Auger decay and radiative decay. ${ }^{13}$ In total, we treat 63 different configurations states, up to $\mathrm{Ne}^{10+}$ and simulate the chaotic intensity profile of the SASE XFEL radiation with a Monte Carlo method, which determines random SASE intensity profiles, given an average pulse duration, the photon energy and the bandwidth. ${ }^{24}$ We have supposed a relative SASE bandwidth of $\Delta \omega_{P} / \omega_{P}=4 \times 10^{-4}$, in accordance to design parameters of the LCLS. ${ }^{3}$ This gives a coherence time $\tau_{c}=\sqrt{\pi} / \Delta \omega_{P}$ of $\sim 3$ fs - the average duration of a coherent SASE spike.

${ }^{*}$ We estimated the electron-ion collision time $\tau$ by a simple geometrical approach, ${ }^{22} \tau \sim\left(n_{e} \pi a_{0}^{2}\right)^{-1}\left(8 K T_{e} / \pi m_{e}\right)^{-1 / 2}$, where $n_{e}$ is the electron density, $a_{0}$ the Bohr radius, $m_{e}$ the electron mass, $K$ the Boltzmann constant and $T_{e}$ the electron temperature. In case of neon pumped with $1 \mathrm{keV}$ photon energy, we will deal with two sort of electron temperatures: slow photoelectrons of around $100 \mathrm{eV}$ energy and hot Auger electrons with energies of approximately $800 \mathrm{eV}$. Supposing a gas density of $10^{19}$ atoms $/ \mathrm{cm}^{3}$, the electron-ion collision time for the photoelectrons will be around $\tau \sim 800$ fs, that for Auger electrons $\tau \sim 570 \mathrm{fs}$, i.e. long compared to the XFEL pulse duration and the pumping process 


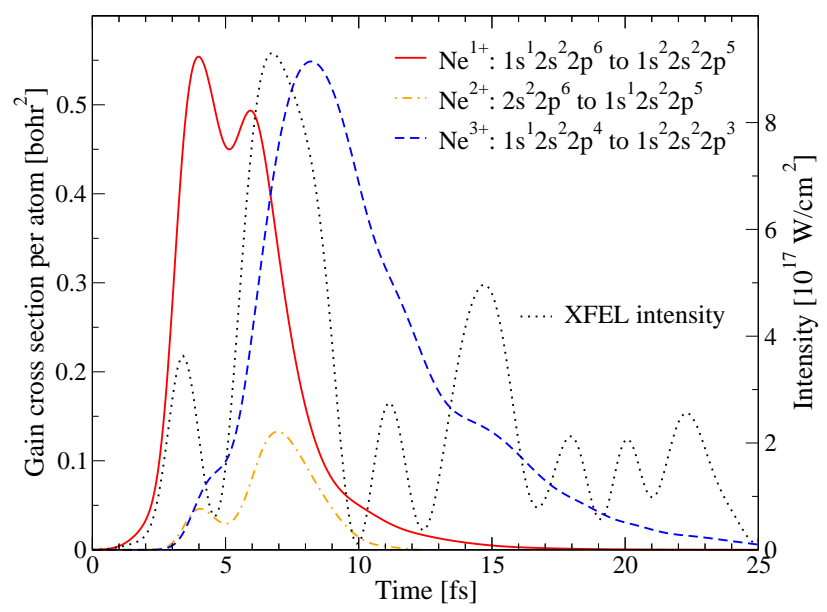

Figure 1. Small-signal gain cross section per atom for the strongest transitions in singly, doubly and triply ionized neon for a sample XFEL pulse of $1 \mathrm{keV}$ photon energy. For the XFEL pulse we assumed $5 \times 10^{12}$ photons, a pulse duration of $100 \mathrm{fs}$ and a focus spot diameter of $2 \mu \mathrm{m}$.

Fig. (1) shows the temporal profile of the small-signal gain cross sections per atom in atomic units ${ }^{\dagger}$ for a sample XFEL pulse (black dotted line) of $1 \mathrm{keV}$ photon energy. The parameters of the pulse are given in the figure caption. Shown are the highest gain cross sections for different charge states of neon. The gain maxima are temporally correlated with the maxima of the intensity spikes of the XFEL pump and are separated by a few femtoseconds. The first XFEL spike ionizes an electron from the K-shell, creating a population inversion between the the levels $1 \mathrm{~s}^{1} 2 \mathrm{~s}^{2} 2 \mathrm{p}^{6}$ and $1 \mathrm{~s}^{2} 2 \mathrm{~s}^{2} 2 \mathrm{p}^{5}$ of $\mathrm{Ne}^{1+}$ [corresponding to the red solid line in Fig. (1)]. The width of the $\mathrm{Ne}^{1+}$ transition line is determined by the Auger lifetime of the core hole, which is 2.75 femtoseconds. The probability to produce a double core-hole in the K-shell by another photoionization event, before Auger decay of the 1s hole takes place, is small. Hence, the gain cross-section of the $\mathrm{Ne}^{2+}$ transition $2 \mathrm{~s}^{2} 2 \mathrm{p}^{6}$ to $1 \mathrm{~s}^{1} 2 \mathrm{~s}^{2} 2 \mathrm{p}^{5}$ is relatively small (orange dash-dotted line). The dominant Auger decay channel of the core-excited $\mathrm{Ne}^{1+}$ is to $\mathrm{Ne}^{2+} 2 \mathrm{~s}^{2} 2 \mathrm{~s}^{2} 2 \mathrm{p}^{4}$. This state is subsequently core ionized by the XFEL pulse, giving rise to the second strongest transition from $\mathrm{Ne}^{3+} 1 \mathrm{~s}^{1} 2 \mathrm{~s}^{2} 2 \mathrm{p}^{4}$ (blue dashed line). For the particular sample pulse chosen, the peak gain-cross sections for the $\mathrm{Ne}^{1+}$ and $\mathrm{Ne}^{3+}$ transition are of similar size. This implies, that it is possible to saturate both lasing transitions. This observation will be corrobated by a one-dimensional self-consistent gain calculation, presented in the next section.

Due to the chaotic nature of the XFEL pump, small-signal gain cross sections and hence the output of the XRL will vary on a shot-to-shot basis. We therefore averaged the cross sections over an ensemble of random pulses. The general trend of the averaged temporal gain-profile is similar to that of the single-shot profile. The average values and standard deviations of the peak gain cross section, as well as their duration at FWHM is given in Tables 1 and 2 for 1 and $1.4 \mathrm{keV}$ pumping photon energy respectively.

Pumping with $1.4 \mathrm{keV}$ photon energy, long lived helium and hydrogen-like lasing transitions in neon become accessible. These transitions have extremely narrow natural widths in the order of $10^{-6}-10^{-5}$. This opens the pathway to a XRL source of increased longitudinal (temporal) coherence length $L_{L}$, which can be estimated by $L_{L}=\lambda^{2} / \Delta \lambda$. In case of the hydrogen-like transition in neon at $\lambda=1.2 \mathrm{~nm}$ this would result in a coherence length $L_{L}=300 \mu \mathrm{m}$, or a longitudinal coherence time of approximately a picosecond, i.e. the pulses created would have full temporal coherence. This would be a great improvement to SASE FEL pulses, albeit with a drop in total power and peak intensity. The XRL amplification is limited by the saturation intensity, which is defined by the

\footnotetext{
${ }^{\dagger}$ A common quantity for XRLs is the so called gain coefficient $G=g \cdot n$, usually measured in units of $\mathrm{mm}^{-1} \mathrm{or} \mathrm{cm}^{-1}$. At atomic densities of $n=10^{18} \mathrm{~cm}^{-3}$ the gain coefficient (in $\mathrm{mm}^{-1}$ ) is related to the gain cross section (in bohr ${ }^{2}$ ) by $G=2.8 \mathrm{~g}$. The XRL will saturate at gain-length products of typically $G \cdot L \sim 20 .^{25}$ For typical gain-cross sections in the order of one a.u.; the proposed XRL will hence saturate after a few $\mathrm{mm}$ interaction length, at gas densities of $n=10^{18} \mathrm{~cm}^{-3}$.
} 
intensity, at which the rate of stimulated emission equals the exit rate of the upper lasing state and given by

$$
I_{s a t}=\frac{\Delta \omega_{U}}{A_{U \rightarrow L}} \frac{\omega^{3} \Delta \omega}{2 \pi c^{2}}
$$

The width of the lasing line $\Delta \omega$ is dominated by the inverse lifetime of the upper lasing state $\Delta \omega_{U}$. Hence, the saturation flux scales as $\Delta \omega_{U}^{2} / A_{U \rightarrow L}$. For the hydrogen-like transitions, the lifetime of the upper state is relatively long (on the order of $160 \mathrm{fs}$ ), as compared to the Auger-decaying transitions, resulting in a smaller saturation intensity. The saturation intensities for the dominant transitions are listed in tables 1 and 2 .

\section{SELF-CONSISTENT GAIN CALCULATIONS}
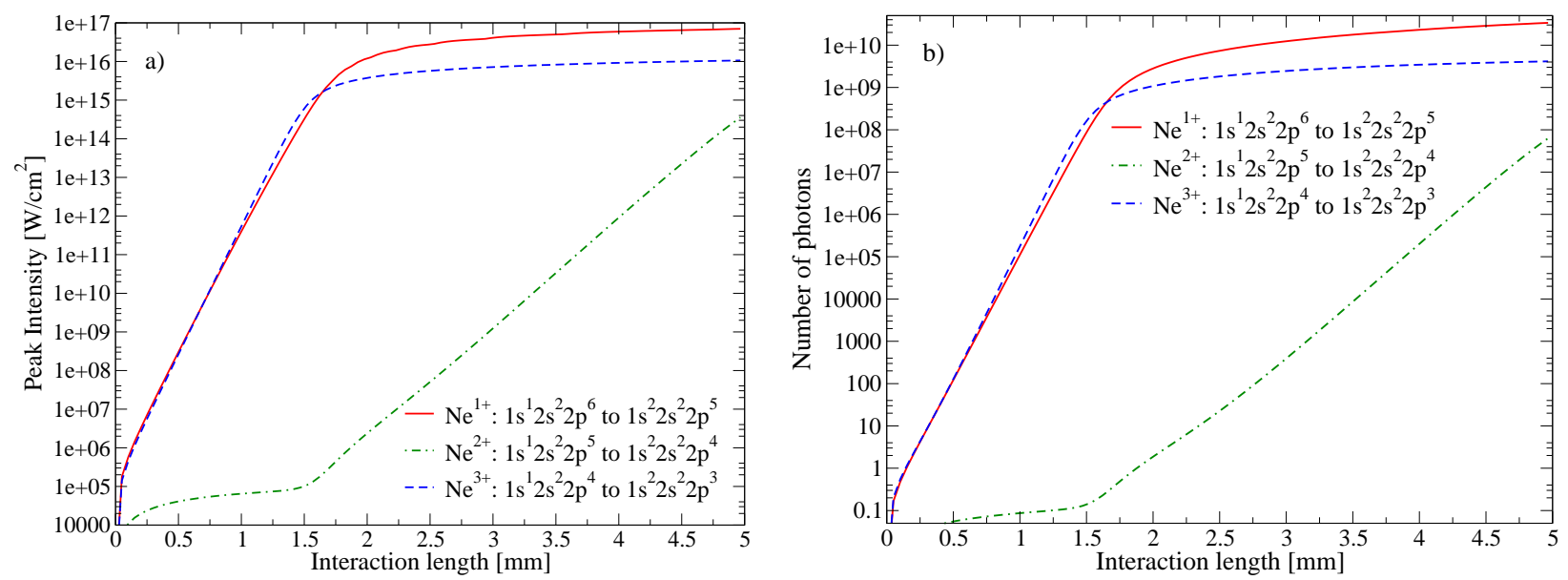

Figure 2. Peak intensity (a) and integrated number of photons (b) of the XRL transitions as a function of the interaction length for the strongest XRL gain of singly, doubly and triply ionized neon, as determined by the one-dimensional selfconsistent gain model for a sample XFEL pulse. The sample XFEL pulse corresponds to the pulse in Fig. 1. We assumed an atomic density of $10^{19}$ atoms $/ \mathrm{cm}^{3}$.

In previous work ${ }^{26}$ we have studied lasing from a single lasing transition corresponding to the highest smallsignal gain cross section. In this contribution, we extended our self-consistent gain model to several lasing states, to see, if saturation of more than one lasing transition is possible, as inferred from the small-signal gain calculations. To simulate the output of the lasing transitions, we apply a one-dimensional model that couples the atomic level kinetics to the laser propagation and amplification. We verified numerically that, due to the longitudinal pumping, lasing occurs only in forward direction. Radiation propagating against the propagation direction of the pump pulse is negligible and will no longer be considered in the following. We treat valence and core ionization by the XFEL pump radiation, spontaneous and stimulated radiative decay and Auger decay. The rate equation for determining the occupation of the upper and lower lasing states $N_{U}^{M}$ and $N_{L}^{M}$ at position $z$ and time $t$, where $M$ denotes the ionic charge state, $U$ and $L$ is an index defining the atomic configuration of the upper and lower lasing states, are determined by:

$$
\begin{aligned}
\frac{d N_{U}^{M}(z, t)}{d t}= & \sum_{i} \sigma_{i}^{v} j(z, t) N_{i}^{M-1}(z, t)+\sum_{i} \sigma_{i}^{c} j(z, t) N_{i}^{M-1}(z, t)-\left[A_{k \rightarrow l}+p_{U}^{A}+\left(\sigma_{U}^{v}+\sigma_{U}^{c}\right) j(z, t)\right] N_{U}^{M}(z, t) \\
& -\sigma^{s e} j_{U}^{X R L}(z, t) N_{U}^{M}(z, t)+\sigma^{a b s} j_{U}^{X R L}(z, t) N_{L}^{M}(z, t) \\
\frac{d N_{L}^{M}(z, t)}{d t}= & \sum_{i} \sigma_{i}^{v} j(z, t) N_{i}^{M-1}(z, t)+\sum_{i} \sigma_{i}^{c} j(z, t) N_{i}^{M-1}(z, t)-\left[\sigma_{L}^{v}-\sigma_{L}^{c}\right] j(z, t) N_{L}^{M}(z, t) \\
& +\sigma^{s e} j_{U}^{X R L}(z, t) N_{U}^{M}(z, t)-\sigma^{a b s} j_{U}^{X R L}(z, t) N_{L}^{M}(z, t)
\end{aligned}
$$




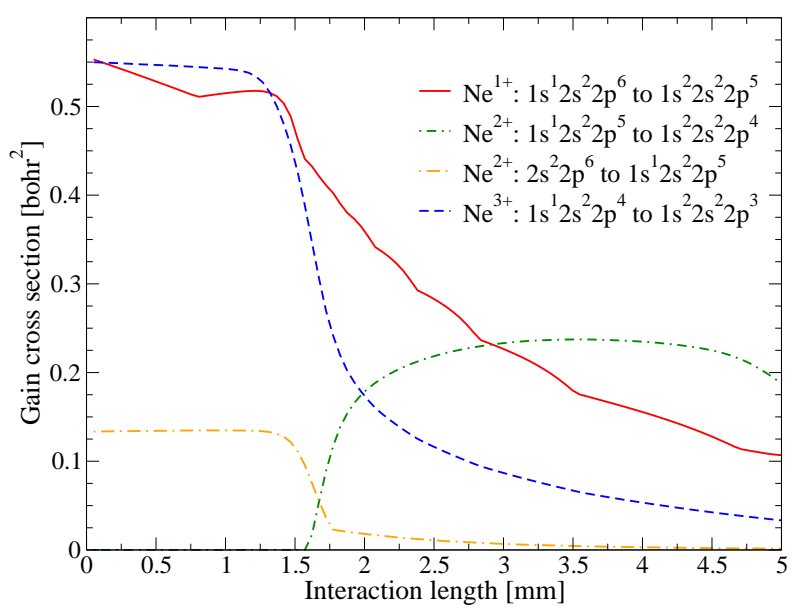

Figure 3. Peak gain cross section of the dominant XRL transitions of singly, doubly and triply ionized neon as a function of the interaction length, as determined from the level occupancies resulting from the one-dimensional self-consistent gain calculation for a sample XFEL pulse. The sample XFEL pulse is the same as in Fig. (1). We assumed an atomic density of $10^{19}$ atoms $/ \mathrm{cm}^{3}$.

where $\sigma_{i}^{v}$ and $\sigma_{i}^{c}$ denote the cross section for valence and core photoionization ${ }^{27}$ respectively for a general state $i$ (not necessary a state giving rise to a lasing transition) with occupation $N_{i}^{M-1}$. The propagating flux of the XFEL pump pulse is denoted by $j(z, t)$ and $p_{U}^{A}$ is the total Auger decay rate of the upper lasing state. Absorption of the XFEL pump $j(z, t)$ is taken into consideration, by an additional differential equation coupled to Eqs. (5) and (6). The second row of Eqs. (5) and (6) corresponds to the stimulated absorption and emission of the forward propagating XRL flux $j_{U}^{X R L}$. The XRL flux from transition $U \rightarrow L$ is determined by

$$
\frac{d j_{U}^{X R L}}{d t}=j_{U}^{X R L}(z, t) c n_{A}\left[\sigma^{s e} N_{U}^{M}(z, t)-\sigma^{a b s} N_{L}^{M}(z, t)\right]+\frac{\theta(z)}{4 \pi} A_{U \rightarrow L} N_{U}^{M}(z, t) n_{A} c-c \frac{d j_{U}^{X R L}}{d z}
$$

where $\theta(z)=2 \pi\left[1-(L / 2-z) / \sqrt{r^{2}+(L / 2-z)^{2}}\right]$ is a geometrical acceptance angles, allowing propagation in forward direction, $n_{A}$ is the atomic density and $L$ the interaction length and $r$ is the focal radius.

Figure 2 shows the results of the self-consistent gain calculations for the sample pulse shown in Figure 1. Shown are the total number of photons in the XRL radiation (b) and the peak-intensity of the XRL pulse (a) for the dominant lasing transitions of singly, doubly and triply ionized neon as a function of the interaction length. We assumed a gas density of $10^{19} \mathrm{~cm}^{-3}$. The $\mathrm{Ne}^{1+} 1 \mathrm{~s}^{1} 2 \mathrm{~s}^{2} 2 \mathrm{p}^{6}$ to $1 \mathrm{~s}^{2} 2 \mathrm{~s}^{2} 2 \mathrm{p}^{5}$ transition (850 eV, red solid line) and $\mathrm{Ne}^{3+} 1 \mathrm{~s}^{1} 2 \mathrm{~s}^{2} 2 \mathrm{p}^{4}$ to $1 \mathrm{~s}^{2} 2 \mathrm{~s}^{2} 2 \mathrm{p}^{3}$ (862 eV, blue dashed line) have similar initial small-signal gain (see Fig. 1). Both lines amplify exponentially with the same growth rate and start to saturate after $\sim 1.5 \mathrm{~mm}$. At the exit of the plasma column there are $\sim 3 \times 10^{10}$ photons in the $\mathrm{Ne}^{1+}$ transition, $\sim 4 \times 10^{9}$ photons in the $\mathrm{Ne}^{3+}$ transition. As can be seen in table 1 , the $\mathrm{Ne}^{3+}$ transition has slightly lower saturation intensity and the gain curve flattens out at a shorter interaction length. Once saturation sets in, the level occupancies change dramatically, i.e. the stimulated emission becomes faster than the Auger-decay. In this regime, the amplification is no longer determined by the small-signal gain cross sections. Lines, that initially had no positive gain cross section suddenly show finite gain and amplify exponentially. This can be seen in the kick-off of the $\mathrm{Ne}^{2+} 1 \mathrm{~s}^{1} 2 \mathrm{~s}^{2} 2 \mathrm{p}^{5}$ to $1 \mathrm{~s}^{2} 2 \mathrm{~s}^{2} 2 \mathrm{p}^{4}$ transition (855 eV, green dash-dotted line). The peak gain-cross section for the dominant lasing lines is depicted in Figure 3 as a function of the interaction length and reflects the change of the level occupancies. The peak gain for the $\mathrm{Ne}^{1+}$ and $\mathrm{Ne}^{3+}$ transition are of similar magnitude at the start of the interaction medium. At $z=1.5$ the ampflification of the $\mathrm{Ne}^{1+}$ and $\mathrm{Ne}^{3+}$ transitions saturates and the gain cross sections of the $\mathrm{Ne}^{2+}$ transitions change dramatically. The gain of the initially dominant transition of the double-core excited $\mathrm{Ne}^{2+} 2 \mathrm{~s}^{2} 2 \mathrm{p}^{6}$ drops. The stimulated emission from the $\mathrm{Ne}^{1+}$ transition happens faster than the creation of a second core-hole and Auger decay of the $\mathrm{Ne}^{1+} 1 \mathrm{~s}^{1} 2 \mathrm{~s}^{2} 2 \mathrm{p}^{6}$ state, mainly populating the $\mathrm{Ne}^{1+} 1 \mathrm{~s}^{2} 2 \mathrm{~s}^{2} 2 \mathrm{p}^{5}$ state. This state subsequently core-ionizes, giving rise to the population inversion of the $\mathrm{Ne}^{2+} 1 \mathrm{~s}^{1} 2 \mathrm{~s}^{2} 2 \mathrm{p}^{5}$ to $1 \mathrm{~s}^{2} 2 \mathrm{~s}^{2} 2 \mathrm{p}^{4}$ transition. The peak 


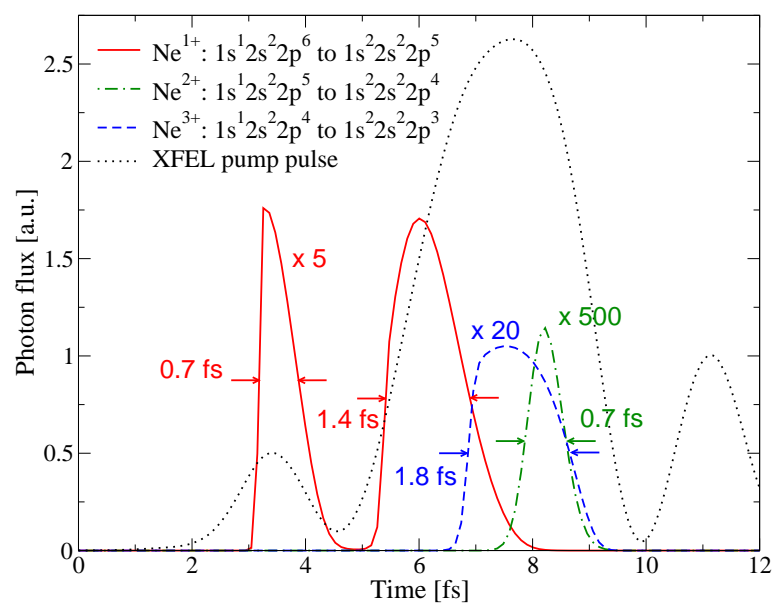

Figure 4. Output flux in a.u. of the dominant XRL lines and the transmitted XFEL pump pulse as a function of time. For better visibility, the XRL fluxes have been scaled, as indicated by the $\times$ factors.

gain of the $\mathrm{Ne}^{1+}$ transition shows a slightly bumpy, non-monotonic structure, which is due to the temporal double-hump structure in the small-signal gain (see Figure 1) and the absorption of the pumping XFEL pulse.

The temporal output profiles of the different XRL lasing lines are shown in Fig. 4, along with the transmitted XFEL pulse. For the particular XFEL sample pulse, the $\mathrm{Ne}^{1+}$ transition at $850 \mathrm{eV}$ photon energy (red solid line) results in two pulses of $0.7 \mathrm{fs}$ and $1.4 \mathrm{fs}$ pulse duration (FWHM), separated by $3 \mathrm{fs}$. The $\mathrm{Ne}^{3+}$ transition at 862 has a pulse duration of $1.8 \mathrm{fs}$ and a flux that is a factor of 4 smaller than the $\mathrm{Ne}^{1+}$ transition. We emphasize the fact that these results are for a particular sample shot. Pulse duration, the number of pulses (typically 1-2) at a given wavelength and their relative peak intensities will vary on a shot-to-shot basis. The average small-signal gains of the $\mathrm{Ne}^{1+}$ and $\mathrm{Ne}^{3+}$ transitions are of comparable size, but they vary on a shot-to-shot basis. It is therefore possible, that for a subset of XFEL pulses, the $\mathrm{Ne}^{3+}$ lasing line cannot be saturated. The reason for this is that once the $\mathrm{Ne}^{1+}$ saturates, the gain of the $\mathrm{Ne}^{3+}$ transitions drops, due to the change in level kinetics. In cases, where the initial small-signal gain of $\mathrm{Ne}^{3+}$ was considerably smaller than that of $\mathrm{Ne}^{1+}$, the amplification of $\mathrm{Ne}^{3+}$ is quenched as soon as saturation of $\mathrm{Ne}^{1+}$ sets in, and the $\mathrm{Ne}^{3+}$ will have small output power with an intensity smaller than the saturation intensity. The lasing transition resulting from the $\mathrm{Ne}^{2+} 1 \mathrm{~s}^{1} 2 \mathrm{~s}^{2} 2 \mathrm{p}^{5}$ state has a duration of $0.7 \mathrm{fs}$. Although its peak intensity is a factor of 100 smaller than that of the $\mathrm{Ne}^{1+}$ line, there are still $\sim 6 \times 10^{7}$ photons contained in this pulse. This total number of photons is comparable to sub-femtosecond VUV sources composed of higher-harmonic radiation of optical laser pulses and hence can serve as a probe pulse in a multi-color x-ray pump-probe application of our proposed XRL.

\section{CONCLUSIONS}

In this paper we presented detailed self-consistent gain calculations of a recently proposed inner-shell atomic XRL pumping scheme using SASE XFEL radiation. ${ }^{26}$ We discussed results for neon in a parameter regime that corresponds to planned experiments at the LCLS. As inferred from the small-signal gain cross sections for neon, it is possible to subsequently saturate more than one lasing transition, pertaining to different ionic charge states, thereby producing a series of femtosecond x-ray pulses of different wavelengths, at intensities comparable to that of the pumping XFEL. The pulse structures, durations and intensities will vary, however, on a shot-to-shot basis, due to the chaotic nature of the SASE XFEL pump pulse. The proposed inner-shell ionization XRL pumping scheme could open pathways towards multi-color non-linear pump-probe experiments in the x-ray regime.

\section{ACKNOWLEDGMENTS}

Work supported by the U.S. Department of Energy under contract No. DE-AC52-07NA27344; support from the Laboratory Directed Research and Development Program at LLNL is also acknowledged. 


\section{REFERENCES}

1. D. L. Matthews et al., "Demonstration of a soft x-ray amplifier," Phys. Rev. Lett. 54, p. 110, 1985.

2. B. J. MacGowan et al., "Short wavelength x-ray laser research at the lawrence livermore national laboratory," Phys. Fluids B 4, p. 2326, 1992.

3. J. Arthur et al., Linac Coherent Light Source (LCLS) Conceptual Design Report. Stanford Linear Accelerator Center, SLAC report 593, 2002.

4. P. Emma for the LCLS commisioning team, "First lasing of the lcls x-ray fel at 1.5 a," tech. rep., SLAC, Stanford, 2009.

5. R. Bonifacio, C. Pellegrini, and L. M. Narducci, "Collective instabilities and high-gain regime in a free electron lasers," Opt. Commun. 50, p. 373, 2002.

6. E. L. Saldin, E. A. Schneidmiller, and M. V. Yurkov, "Statistical properties of radiation from vuv and x-ray free electron laser," Opt. Commun. 148, p. 383, 1998.

7. H. Wabnitz, A. de Castro, P. Gürtler, T. Laarmann, W. Laasch, J. Schulz, and T. Möller, "Multiple ionization of rare gas atoms irradiated with intense vuv radiation," Phys. Rev. Lett. 94, p. 023001, 2005.

8. R. Santra and C. Greene, "Multiphoton ionization of xenon in the vuv regime," Phys. Rev. A 70, p. 053401, 2004.

9. A. A. Sorokin, M. Wellhofer, S. V. Bobashev, K. Tiedtke, and M. Richter, "X-ray-laser interaction with matter and the role of multiphoton ionization: Free-electron-laser studies on neon and helium," Phys. Rev. A 75, p. 051402(R), 2007.

10. M. G. Makris and P. Lambropoulos, "Theoretical interpretation of multiphoton ionization of neon by softx-ray intense radiation," Phys. Rev. A (77), p. 023401, 2008.

11. M. G. Makris and P. Lambropoulos, "Reexamination of multiphoton ionization of xenon under 12.7-ev radiation," Phys. Rev. A 77, p. 023415, 2008.

12. M. G. Makris, P. Lambropoulos, and A. Mihelic, "Theory of multiphoton multielectron ionization of xenon under strong 93-ev radiation," Phys. Rev. Lett. 102, p. 033002, 2009.

13. N. Rohringer and R. Santra, "X-ray nonlinear optical processes using a self-amplified spontaneous emission free-electron laser," Phys. Rev. A 76, p. 033416, 2007.

14. P. Emma et al., "Femtosecond and sub-femtosecond x-ray pulses from a self-amplified spontaneous-emissionbased free-electron laser," Phys. Rev. Lett. 92, p. 074801, 2004.

15. Y. Ding et al., "Measurements and simulations of ultralow emittance and ultrashort electron beams in the linac coherent light source," Phys. Rev. Lett. 102, p. 254801, 2009.

16. N. R. Thompson and B. NcNeil, "Mode locking in a free-electron laser amplifier," Phys. Rev. Lett. 100, p. 203901, 2008.

17. Y. Wang et al., "Phase-coherent, injection-seeded, table-top soft-x-ray lasers at $18.9 \mathrm{~nm}$ and $13.9 \mathrm{~nm}$," Nature photonics 2, p. 94, 2008.

18. J. Zhao et al., "X-ray lasers from inner-shell transitions pumped by the free-electron laser," Optics Express 16, p. 3546, 2008.

19. K. Lan, E. Fill, and J. Meyer-Ter-Vehn, "Photopumping of xuv lasers by xfel radiation," Laser Part. Beams 22, p. 261, 2004.

20. S. Jacquemot, K. T. Phuoc, A. Rousse, and S. Sebban, "Modelling of an inner-shell photo-ionization x-ray laser using a recently demonstrated betatron source," in X-ray Lasers 2006, Springer, 2007.

21. M. A. Duguay and G. P. Rentzepis, "Some approaches to vacuum uv and x-ray lasers," Appl. Phys. Lett 10, p. 350, 1967.

22. T. S. Axelrod, "Inner-shell photoionization-pumped x-ray lasers. sulfur," Phys. Rev. A 13, p. 376, 1976.

23. R. A. London, "Development of coherent x-ray lasers," Phys. Fluids B 5, p. 2707, 1993.

24. G. Vannucci and M. C. Teich Appl. Opt. 19, p. 548, 1980.

25. R. C. Elton, X-ray lasers, Academic Press, Inc., 1990.

26. N. Rohringer and R. London, "Atomic inner-shell x-ray laser pumped with an x-ray free electron laser," Phys. Rev. A 80, p. 013809, 2009.

27. Los Alamos National Laboratory Atomic Physics Codes. http://aphysics2.lanl.gov/cgibin/ION/runlanl08a.pl. 